\title{
Penser la sexualité et les rapports sociaux de sexe : perspectives critiques. Dialogue entre deux disciplines
}

\section{Marianne Blidon et Natacha Chetcuti}

\section{CpenEdition}

Journals

Édition électronique

URL : http://journals.openedition.org/cdg/1805

DOI : $10.4000 /$ cdg. 1805

ISSN : 2107-7266

Éditeur

UMR 245 - CESSMA

Référence électronique

Marianne Blidon et Natacha Chetcuti, «Penser la sexualité et les rapports sociaux de sexe:

perspectives critiques. Dialogue entre deux disciplines », Carnets de géographes [En ligne], 1 | 2010, mis en ligne le 01 octobre 2010, consulté le 14 septembre 2020. URL : http://journals.openedition.org/cdg/ 1805

\section{(c) (†) $\odot$}

La revue Carnets de géographes est mise à disposition selon les termes de la Licence Creative Commons Attribution - Pas d'Utilisation Commerciale - Pas de Modification 4.0 International. 


\title{
Penser la sexualité et les rapports sociaux de sexe : perspectives critiques Dialogue entre deux disciplines
}

\author{
Marianne Blidon \\ MCF (géographe), IDUP-Paris 1 \\ Natacha Chetcuti \\ Chercheur (sociologue et anthropologue), INSERM
}

Nous avons pris la proposition d'écrire un article à deux voix pour le premier numéro des Carnets du géographe comme une invitation à échanger et à confronter nos expériences disciplinaires autour des questions sexuelles et des rapports sociaux de sexe. La ligne éditoriale de la revue et son positionnement dans le champ nous ont incitées à assumer un style d'écriture que nous avons souhaité - sans peut-être toujours y parvenir faute d'être totalement familières avec ce type d'exercice - plus personnel, réflexif et impliqué. C'est pourquoi nous avons opté pour la forme moins académique d'un dialogue structuré autour de questions qui nous paraissaient centrales ou heuristiques. Cette forme dialoguée, ponctuée par des questions, présente l'avantage de mieux différencier nos positions respectives et d'assumer plus facilement le «je». Ce dialogue a pour partie eu lieu, pour partie était prolongé par des échanges épistolaires. Il se structure autour de trois axes : la construction de nos objets de recherche, l'articulation entre genre, sexualité et d'autres formes de rapport de pouvoir et nos parcours académiques.

Si les catégories d'hétérosexualité et d'homosexualité font désormais partie du vocabulaire académique, leur articulation avec une analyse des rapports sociaux de sexe semble s'effacer. Ce refus de prise en considération des effets de ces catégorisations, articulées à celles des rapports sociaux d'âge, de race et de classe sociale, risque de conduire à la production d'objets désintriqués des rapports de domination inhérents à la structure sociale. Ayant en commun de travailler sur les catégories d'homosexualité et de genre, nous avons été amenées à rencontrer cette question à partir de champs de recherche différents : la géographie de l'homosexualité, principalement masculine, pour l'une, et la sociologie du genre articulée à celle du lesbianisme pour l'autre. Nous avons souhaité ouvrir la discussion sur la place du genre dans nos parcours de recherche et les problèmes que pose son articulation à d'autres catégories d'analyse comme le sexe et la sexualité. Il s'agira également de mettre en évidence les éventuelles résistances disciplinaires et institutionnelles à conférer une légitimité aux homosexualités en tant qu'objet d'étude en ne les limitant pas aux seules pratiques sexuelles. 


\section{La construction d'un objet de recherche}

\section{Comment nous sommes-nous saisi des questions sur l'homosexualité dans nos travaux?}

NC : Pour ma part, j'en suis venue à travailler sur les catégorisations de genre et de sexualité à travers une analyse des expériences lesbiennes par la lecture de textes théoriques, il y a maintenant plus de dix ans. C'était à la fin des années 1990 que, lors de mon cursus de sociologie, j'ai rencontré les textes de Nicole Claude Mathieu (anthropologue) et ceux de Monique Wittig. Trois textes ont été particulièrement fondateurs : «Identité sexuelle/sexuée/de sexe ? Trois modes de conceptualisation du rapport entre sexe et genre », de Nicole Claude Mathieu (1991), On ne naît pas femme et La pensée Straight de Monique Wittig (1980a, 1980b). Ces trois textes émanant de champs disciplinaires différents, ont guidé mes premiers pas vers une dé/naturalisation des catégorisations de sexe. En effet, dans son article Nicole-Claude Mathieu s'interroge sur les modes de conceptualisation du rapport entre sexe et genre dans les sociétés occidentales, tant au niveau des représentations sociales que des relations sociales. Son classement s'établit à deux niveaux : d'une part, la conceptualisation abstraite et théorique, et d'autre part, les pratiques, les représentations sociales ainsi que la stratégie mise en œuvre par les groupes sociaux spécifiques à chaque société. Les deux articles de Monique Wittig, quant à eux, analysent la dimension politique de l'hétérosexualité et critiquent le présupposé de la différence des sexes qui structure la pensée de la différence en donnant un statut inné et a priori à l'hétérosexualité. Elle démontre que cette différence sexuelle, qui émanerait du corps, n'est que la justification d'une idéologie qui opère une classification arbitraire structurant le rapport de force inégalitaire entre les hommes et les femmes. La conséquence d'une pensée naturalisante tenant pour logique et inévitable la distinction entre masculin et féminin est d'entretenir le déséquilibre social de la répartition du pouvoir entre hommes et femmes. Pour Wittig, la distinction entre homosexualité et hétérosexualité dépend de la distinction construite entre homme et femme et féminin/masculin, cette distinction étant à la base de la société hétérosexuelle.

MB : Tu n'as pas fait référence à la formule de Wittig: «les lesbiennes ne sont pas des femmes » dont tu parles dans ton article (Chetcuti, 2009).

NC : Pour Wittig, la catégorie «femme » n'a de sens que dans ce système, et c'est pour cela qu'elle lance l'énoncé aujourd'hui bien connu : «les lesbiennes ne sont pas des femmes »; ce qui ne veut pas dire pour autant qu'elles ne sont pas des femmes sur le plan de l'appropriation collective (inégalité de salaires, violences sexuelles, etc.). Mais selon Wittig, elles ne sont pas des femmes dans le sens où elles échappent à l'appropriation privée (par le fait de ne pas vivre de relations sexuelles avec des hommes). Le concept d'appropriation privée en relation avec l'appropriation collective est tiré des travaux de Colette Guillaumin (1992). Ses analyses ont montré que la force des rapports sociaux vient de cette idéologie inhérente aux rapports sociaux eux-mêmes et à la force discursive de la bicatégorisation des sexes. Pour Colette Guillaumin, il faut expliquer la question de la domination masculine sous une forme plus globale car c'est un ensemble de rapports qui fait système. Cette sociologue introduit la notion d'appropriation collective et privée de la classe de sexe femme : le sexage par analogie avec esclavage (elle montre dans son ouvrage L'idéologie raciste (2002) que le concept de race dans son acception moderne n'existait pas avant l'esclavage). Le sexage désigne l'appropriation physique et mentale d'une classe entière au-delà de la force de travail. L'appropriation privée des femmes se manifeste dans le mariage ou dans toute autre relation contractualisée. Elle comporte l'appropriation du temps et des produits du corps, l'obligation sexuelle et la charge physique des membres du groupe, de la famille. 
MB : Et qu'est-ce que ces travaux t'ont apporté ?

NC : L'ensemble de ces travaux m'a permis de ne plus envisager les catégories de sexe et de genre, mais m'ont bien plutôt incitée à les insérer dans un système de pouvoir qui se traduit aussi bien dans les pratiques sociales que dans les productions «scientifiques ». Pour moi, la rencontre de ces textes a révélé un vrai tournant épistémologique, et c'est ainsi que progressivement j'ai défini mon sujet de thèse, qui a porté sur l'analyse de la construction du lesbianisme dans le contexte contemporain à travers trois dimensions : l'auto-nomination de soi, les modalités de couple et le script sexuel ${ }^{1}$. Ma question était alors très fortement liée à la proposition théorique de Wittig : à savoir, en quoi les lesbiennes pouvaient-elles interroger la catégorie «femme»?

En outre, ces travaux, comme l'ensemble des conceptualisations féministes des années 1970 et 1980, restent aujourd'hui encore à mon sens une base fondamentale en sciences humaines pour appréhender le rapport entre sexe et genre et l'articulation avec «l'orientation sexuelle ». Ces innovations théoriques portant sur les catégories de sexe et de sexualité ont conduit à interroger ce qui semblait alors relever d'un ordre "naturel ». L'usage du terme " genre » et les différentes théorisations du sexe ont ainsi permis de mettre l'accent sur tout un système de relations qui inclut le sexe, mais ne sont pas directement déterminées par lui ni ne déterminent la sexualité dans sa totalité.

MB : Dans ce cadre, comment as-tu construit ton terrain?

NC : J'ai constitué mon terrain dès mon année de maîtrise puisqu'à l'époque, je travaillais sur les représentations de soi (mise en scène de soi, usage vestimentaire, etc.) des lesbiennes en milieu militant, et je l'ai continué lors de mon DEA, dans lequel j'ai davantage produit un état des lieux de la littérature théorique destinée à préparer l'entrée en doctorat. Dans mon travail de thèse, j'ai donc mobilisé un terrain déjà connu, car sans y participer activement en tant que militante, j'étais un membre visible de la communauté. Ma position $d^{\prime}$ insider $^{2} \mathrm{~m}$ 'a facilité l'entrée dans plusieurs groupes et permis de m'engager dans les entretiens assez facilement. De plus, alors que je n'étais pas partie prenante en tant qu'acteur politique, j'étais déjà intégrée en tant que chercheur : la publication d'un certain nombre d'ouvrages collectifs, dont celui avec Claire Michard (2003), Lesbianisme et féminisme : histoires politiques, m'avait donné un «label» de chercheur, garantissant une confiance de la part des membres de ma communauté. Toutefois, je dois préciser ici que j'ai opté pour un choix : celui de ne pas faire d'interview auprès de lesbiennes proches de mon réseau social (amical ou professionnel), ce qui me garantissait la pratique du regard éloigné sur les données recueillies.

\footnotetext{
${ }^{1}$ L'auto-nomination (c'est-à-dire sur les définitions que chaque individu donne à son orientation sexuelle) appréhendé en tant que processus permet de saisir le cheminement permettant aux interviewées de se nommer. Le fait de demander aux personnes comment elles se définissent du point de vue de l'orientation sexuelle permet de prendre connaissance des processus d'intégration sociale et de leur part d'autonomie à l'intérieur de ces contraintes. De plus, si l'on considère que la sexualité est prise dans un jeu entre attirance, expérience pratique et auto-identification de soi, s'arrêter sur les définitions que chacune donne à ses pratiques et relations sexuelles est particulièrement révélateur des différentes représentations et statuts de l'homosexualité et de l'hétérosexualité. Le terme de script renvoie au synopsis utilisé dans le cinéma et la télévision ; il permet de distinguer le découpage, les séquences et les dialogues qui composent un scénario sexuel. Cette notion, forgée par Gagnon et Simon en 1973, a été mise au point, à ses débuts, pour analyser les représentations sexuelles explicites de la pornographie.

${ }^{2}$ La position d'insider signifie être membre du groupe étudié, elle s'oppose à la notion d'outsider. Ces notions issues de la sociologie interactionniste ont été largement débattues à leurs débuts dans les travaux entre autres d'Howard Becker (1985).
} 
MB : De ce point de vue, je suis dans une situation et dans une démarche inverse. Je suis dans une position de double outsider [femme et hétérosexuelle], j'y reviendrai. Et ce ne sont pas des textes théoriques qui m'ont amené à travailler sur le thème de l'homosexualité et cela pour différentes raisons. L'une de ces raisons est disciplinaire. Depuis les géographes marxistes et à l'exception des modélisateurs, peu de géographes ont une filiation théorique forte appuyant une démarche déductive. De façon réductrice, on peut dire que la géographie est une science sociale qui repose principalement sur des pratiques de terrain ou sur le traitement de données que ce soit en géographie physique ou humaine. Les références théoriques aux sociologues que ce soit Pierre Bourdieu par exemple dont les travaux sont fréquemment cités en géographie sociale par les géographes français ${ }^{3}$ ou des philosophes comme Foucault ${ }^{4}$, Deleuze ${ }^{5}$ et Lefebvre $^{6}$ qui sont des références incontournables de la géographie radicale britannique et américaine, viennent davantage éclairer l'analyse ou permettre la montée en généralité via l'usage des notions comme l'habitus, le capital, la discipline, l'espace lisse et l'espace strié, l'espace social, etc. La géographie s'appuie traditionnellement sur une démarche inductive et empirique à l'inverse de la sociologie hypothético-déductive. Ma démarche a donc été très empirique : je suis partie d'un espace «le quartier gay du Marais » et d'une population « les gays » dont certains chercheurs comme Michael Pollack (1981) présuppose le partage d'un mode de vie urbain. Toute ma démarche a consisté à interroger la pertinence de cet objet et de cette catégorie. C'est très tardivement et partiellement que j'ai relié mes travaux aux questions de rapports sociaux de sexe.

NC : Mais ce n'est pas seulement une question de démarche disciplinaire ?

MB : Non, effectivement. Cela tient aussi à la genèse de la géographie du genre en France. Celle-ci s'est davantage structurée en référence aux travaux des géographes britanniques comme Linda McDowell, Liz Bondi ou Dorey Masey ${ }^{7}$ qu'aux travaux des féministes françaises, notamment des féministes matérialistes que tu as cité comme Wittig (1980b), Mathieu (1991), Tabet (2004) ou Guillaumin (1992). Ce qui amène, par exemple, Christine Chivallon à affirmer que : « les Feminist Studies ou Gender Studies ou encore Women Studies n'ont pas leur équivalent en France et constituent une spécificité de l'espace académique anglo-américain » $(2001,57)$. Si ce constat s'avère vrai en matière d'espace de diffusion et d'enseignement, il est plus contestable sur le plan de la production, notamment théorique. Ce qui met en relief le faible écho des textes matérialistes en dehors de leur espace d'élaboration thématique et disciplinaire.

Ce fort cloisonnement s'est aussi traduit par une faible collaboration entre géographes et chercheurs d'autres disciplines notamment les sociologues, les anthropologues ou les politistes. Ainsi, les premiers travaux sur l'accès des femmes à l'espace public en France comme l'enquête ENVEFF (Enquête nationale sur les violences envers les femmes en France, 2000) ont été menés par des sociologues et des démographes ${ }^{8}$. Cette absence de coopération

\footnotetext{
${ }^{3}$ Voir notamment les bibliographies de l'ouvrage collectif coordonné par Raymonde Séchet et Vincent Veschambre (2006).

${ }^{4}$ Voir notamment Claude Raffestin (1997) et Juliet Fall (2005).

${ }^{5}$ Voir notamment Mark Bonta et John Protevi (2004).

${ }^{6}$ Voir par exemple Goonewardena Kanishka et ali (2008).

7 Pour s'en convaincre, il suffit de regarder les bibliographies des géographes françaises dans les numéros spéciaux de revues consacrés aux questions de genre. Par exemples, les numéros d'Espace, populations, sociétés dirigés par Dominique Creton Questions de genre (2002) et Espace, genre et sociétés (2004) ou celui de Géographie et cultures dirigé par Francine Barthe et Claire Hancock (2005). Ces dernières citent par exemple : Bondi L. (1993), McDowell L. (1993, 1999) ou Massey D. (1994, 1995).

${ }^{8}$ Pour une présentation de l'enquête voir les travaux de Maryse Jaspard (2007) et pour une synthèse de la question voir l'ouvrage de Marylène Lieber (2008).
} 
s'explique en partie par la fréquentation de réseaux distincts et par la différence d'appartenance disciplinaire. Elle a été renforcée par la rareté des espaces d'échanges pluridisciplinaires diffusant les travaux sur le genre (séminaires, colloques, revues, etc.) au sein de l'espace académique français. Pour ma part, j'ai découvert des théoriciens queer grâce au séminaire de Françoise Gaspard sur La sociologie des homosexualités à l'EHESS en 2003, séminaire auquel tu participais aussi et qui a été un espace d'échanges et de socialisation important pour de nombreux jeunes chercheurs. C'est plus tardivement que j'ai découvert les textes des féministes matérialistes. Pour autant, malgré l'intérêt que je leur porte, je les mobilise peu dans mon travail.

NC : C'est peut-être aussi parce que tu travailles moins sur le genre?

MB : En effet, mon travail porte principalement sur les normes sexuelles beaucoup plus que sur le genre. Le point de départ de mon travail est le hiatus entre un discours anticommunautaire qui posait a priori l'existence d'une communauté gay forclose sur le territoire du Marais et l'observation dans ce quartier parisien d'usages et de relations sociales beaucoup plus riches et complexes que la seule dimension à laquelle on les réduisait. C'était une période où les travaux de géographie travaillaient la relation entre territoire et identité sans avoir intégré les critiques faites à cette notion. C'est l'évidence du lien entre une identité et un quartier qui m'intéressait plus que les rapports sociaux de sexe. Je me suis ensuite intéressée aux effets de l'hétéronormativité ${ }^{9}$ sur les pratiques et aux trajectoires migratoires. Aujourd'hui, j'essaie davantage de penser la géographie des rapports sociaux de sexe. Néanmoins dans ce cadre, le transfert des théories queer ou féministes matérialistes ne se fait pas sans poser des problèmes méthodologiques et épistémologiques.

Il y a dans nos deux parcours deux manières différentes de penser la question de l'homosexualité. Comment ces objets sont-ils apparus dans nos deux disciplines?

NC : En ce qui concerne la sociologie, on peut constater que la question de l'homosexualité a davantage été traitée du point de vue de l'homosexualité masculine et ce dès le début des enquêtes ayant pour objet spécifique l'homosexualité, c'est-à-dire au tournant des années 1990. L'apparition de l'épidémie de sida a, en effet, favorisé un champ d'études des homosexualités masculines le plus souvent restreintes à la question des pratiques sexuelles en lien avec les pratiques préventives à l'égard du sida. Ces enquêtes, en bénéficiant de financements institutionnels, ont permis le développement de cet objet. Toutefois, les lesbiennes ont été les « oubliées » (sic) de ces travaux, tant pour des raisons sociologiques que politiques. Et malgré la publication de recherches pionnières dans les années 1980 et 1990 je pense aux travaux de Marie-Jo Bonnet (1981) en histoire, et à ceux de Claudie Lesselier (1987) et Brigitte Lhomond (1987) en sociologie -, très peu d'études concernant les lesbiennes ont vu le jour en sociologie et en anthropologie dans les années 1990. Des études plus historiques, telles que celles de Florence Binard (2003) sur les lesbiennes dans l'entre-

\footnotetext{
${ }^{9}$ Le terme « normatif » renvoie à la violence ordinaire qu'exercent [perform] certains idéaux de genre ; et c'est un synonyme de « relevant des normes qui gouvernent le genre » (Butler, 2005, 44). Le concept d'hétérosexualité normative (en anglais normative heterosexuality), « désigne le système, asymétrique et binaire, de genre, qui tolère deux et seulement deux sexes, où le genre concorde parfaitement avec le sexe (au genre masculin le sexe mâle, au genre féminin le sexe femelle) et où l'hétérosexualité (procréatrice) est obligatoire, en tout cas désirable et convenable » (Kraus dans Butler, ibid., 24). Dans le cadre de l'hétéronormativité, la régulation du genre peut parfois être une façon de maintenir l'ordre hétérosexuel (symbolique et matériel) et la hiérarchie des sexualités pensée comme universelle et naturelle. Sa puissance réside, comme toute idéologie totalisante, [dans le fait qu'elle] empêche précisément de penser en dehors des cadres de pensée qu'elle institue a priori, « point de vue sur le monde, mais point aveugle de ce point de vue » (Tin, 2003, 208).
} 
deux-guerres ou encore celle de Sandra Boerhinger (2007) sur l'Antiquité ont été conduites, mais en ce qui concerne l'analyse des modes de vie contemporains, il faut attendre les travaux de Virginie Descoutures (2008) sur les mères lesbiennes et mon propre travail de thèse, c'està-dire 2008, pour qu'enfin une « réalité » du lesbianisme émerge en France en tant que sujet d'analyse.

MB : Et comment tu expliques cela?

NC : À mon sens, pour expliquer que ces travaux n'aient pas pu émerger avant, on peut convoquer un ensemble de raisons, fruit des résistances institutionnelles. D'un point de vue sociologique, plusieurs raisons peuvent expliquer la faible prise en compte de travaux sur l'homosexualité féminine. D'abord, la lente progression, en sciences sociales, des études féministes et/ou sur les femmes. En outre, dans ce que l'on a appelé le mouvement d'institutionnalisation du féminisme, dans les années 1980, les objets privilégiés ne concernaient pas les questions d'homosexualité, mais étaient davantage centrés sur la question, entre autres, de l'espace domestique, des inégalités salariales et de la parité dans le champ politique. Déjà à l'époque, les femmes chercheurs, qui se revendiquaient d'un ancrage théorique féministe, avaient beaucoup de mal à trouver une légitimité dans le système académique : parler de lesbianisme, c'était risquer d'en rajouter au discrédit des études sur les femmes. On évoquera ensuite la lente progression, en sciences sociales, des études sur les femmes ou d'études plus proprement féministes. En outre, on mentionnera la force de l'autocensure à l'égard d'un objet de recherche tel que le lesbianisme. De plus, le champ des études sur les sexualités était encore très marginal et peu considéré par les sociologues. Enfin, on peut également souligner que contrairement aux pays anglo-saxons ou outre-Atlantique on se trouve dans un contexte académique et scientifique où les études sur les femmes, sur le genre ou sur les «identités sexuelles » ne sont guère reconnues comme des champs épistémiques et autonomes. Il n'existe en effet pas de départements de feminist studies ou de genders studies ou encore de queer studies ce qui peut sans doute expliquer la faiblesse d'ancrage de ces objets de recherche.

MB : Il me semble que tu n'as pas évoqué des dimensions plus pratiques comme par exemple l'encadrement des thèses...

NC : Effectivement, la faiblesse institutionnelle de ce champ a aussi pour conséquence d'aggraver la situation lorsqu'il s'agit de trouver des personnes susceptibles d'encadrer des thèses. Peu d'enseignants-chercheurs, en tout cas dans les années 1990 et jusqu'au début 2000 étaient prêts à s'engager à encadrer de tels travaux, ce qui n'a pas favorisé l'apparition de cet objet. Il faut d'ailleurs à ce titre rendre hommage à Marie Elisabeth Handman, qui a accepté un certain nombre de thèses traitant, entre autres, de l'homosexualité ; sans ce soutien institutionnel, nombre de thèses n'auraient pas vu le jour, dont la mienne.

Ensuite, ce n'est pas une surprise de constater que l'homosexualité a fait pendant longtemps l'objet de débats essentiellement masculins, ce constat est à l'image des débats publics d'une manière générale, qui sont explicitement ou implicitement traités du point de vue des hommes. La mise en avant d'études sur l'homosexualité masculine au détriment de l'homosexualité féminine tient aussi à l'existence de lois réprimant l'homosexualité visant le plus souvent les pratiques entre hommes. Ce qui ne veut pas dire pour autant que le phénomène de répression de l'homosexualité ne concernerait pas les femmes, mais il fait moins l'objet du débat public quant aux dispositifs légaux. Que ce soit par désintérêt scientifique et politique pour un groupe peu visible et peu puissant, effet de la domination des 
hommes sur les femmes, toutes ces explications peuvent être avancées et ne s'excluent pas ${ }^{10}$. Par ailleurs, on peut se demander si la quasi-inexistence de réflexions et d'études à l'égard des femmes homosexuelles ne peut pas être lue comme une réponse à un contexte social qui peine à intégrer l'homosexualité féminine en tant que sexualité à part entière.

$\mathrm{Au}$ fond, ce qui a permis une relative émergence des sujets traitant du lesbianisme ou en tout cas leur a conféré une plus grande légitimité, ce sont les débats dans les années 1990 autour du pacs. Puis les revendications relatives à la lesboparentalité ont fait émerger un questionnement sur les normes sexuelles et les formes de parenté. S'inscrivent dans cette continuité les travaux sur les rapports sociaux de sexe, ont permis de penser l'usage des catégories homosexualité et hétérosexualité.

MB : En géographie, on retrouve le même phénomène de quasi-absence de la question lesbienne au profit des gays; les lesbiennes étant souvent traitées comme un corolaire des gays sans plus de distinction (Adler Sy, Brenner Johanna, 1992). Cela tient aux dimensions que tu as soulignées et à une plus forte invisibilité (Podmore Julie, 2006). Cependant, les travaux produits dans le cadre de l'ANRS (Agence nationale de la recherche sur le sida) que tu as cité ont eu peu d'échos en géographie et rares sont les géographes à ma connaissance à avoir profité de ces financements. En effet, il y a un clivage important entre les travaux produits par des géographes dans des revues de géographie et les travaux, souvent antérieurs de sociologues comme Michael Pollack (1981), Rommel Mendès Leite (1997, 1998) ou Bruno Proth (2002), mais aussi d'historiens comme Michael Sibalis (1995, 2001) ou d'urbanistes comme Emmanuel Redoutey (2002) dont les objets sont proprement spatiaux (la ville comme fondement du mode de vie gay, l'usage sexuel de l'espace public) avec une forte influence de l'écologie urbaine, mais qui n'ont pas été lus ou reconnus comme tels par les géographes. Ces derniers découvrent d'une certaine manière la question gay avec l'article de Boris Grésillon dans l'Espace géographique (2000), l'ouvrage Géographies anglo-saxonnes (2001) et le débat sur La postmodernité organisé par L'Espace géographique (2004) dans lequel Jean-François Staszak et Béatrice Collignon prennent position en faveur du développement de cette géographie. D'autres travaux suivront: en sociologie, ceux de Baptiste Coulmont (2003) ou de Colin Giraud (2009); en géographie, ceux de Stéphane Leroy (2005), Emmanuel Jaurand (2005), Nicolas Boivin (2008) ou les miens (2007, 2008a, 2008b, 2009). Avec une ambiguité fondamentale, ces travaux ont pour principale référence les géographes britanniques comme Gill Valentine, David Bell ou Jon Binnie qui ont cosigné Mapping Desire (1995) sans en avoir développé les approches postmodernes et situées. Cette équivoque se retrouve aussi dans la géographie espagnole, par exemple dans les travaux de Víctor Fernández Salinas (2007), ou belge avec ceux de Jean-Michel Decroly (2006) qui font de l'analyse spatiale et de la cartographie.

Une autre forme de légitimation et de visibilité de cette thématique a été acquise par le biais des Cafés géographiques ${ }^{11}$ organisés par Gilles Fumey en 2003 ou en 2006, de la journée d'études Sexe de l'espace, sexe dans l'espace ${ }^{12}$ (2007), et des enseignements de master ou de licence qui, de plus en plus nombreux, abordent ces questions.

\footnotetext{
${ }^{10}$ Analysée notamment par Brigitte Lhomond, 1997, «Le sens de la mesure. Le nombre d'homosexuel/les dans les enquêtes sur les comportements sexuels et le statut de groupe minoritaire », Sociologie et sociétés, vol. 29, $\mathrm{n}^{\circ} 1, \mathrm{p} .61-69$.

${ }^{11}$ En 2003 La ville et les communautarismes : le cas du Marais, un « ghetto gay » à Paris ? (http://www.cafegeo.net/article.php3?id_article=270) et en 2006 La planète du sexe $:$ de la prostitution mondialisée au sex shop du coin de la rue (http://www.cafe-geo.net/article.php3?id_article=1019).

12 Boivin Nicolas (dir.), 2008, «Sexe de l'espace, sexe dans l'espace», Les cahiers de l'ADES, n² (http://www.ades.cnrs.fr/spip.php?article492).
} 


\section{Genre, sexualité et autres rapports de pouvoir}

\section{Comment articulons-nous le genre avec les catégories de sexualité, en l'occurrence l'homosexualité ? Comment cela se traduit-il en termes théoriques et méthodologiques?}

NC: Pour ma part, j'ai beaucoup de mal à envisager des travaux sur la sexualité qui n'engagerait pas une analyse en termes de rapports sociaux de sexe. En effet, la sexualité, comme d'ailleurs d'autres cadres d'expérience, est fortement liée aux catégorisations de sexe, dans laquelle la hiérarchisation sociale du genre est encore très présente. Cette articulation se caractérise par l'analyse de la socialisation sexuée et de ses effets sur les discours, les pratiques et la nomination de soi. La sexualité n'est pas une sphère détachée des rapports de pouvoir, c'est une partie de la vie des individus témoignant des configurations sociales de sexe/genre. Par exemple, si l'on sort de l'objet même «sexualité », comment peut-on penser qu'un espace social est neutre ? Ce dernier est au contraire régi par les rapports sociaux de sexe, de race, de classe, d'âge, etc. On agit en fonction de ces critères, en fonction des assignations normatives et des différents statuts sociaux. Selon les contextes où l'on se trouve, telle ou telle autre dimension va plus ou moins être active. Si je prends mon terrain, par exemple, je me suis rendue compte qu'avoir des relations socio-sexuelles avec des femmes ne suffisait pas pour s'auto-définir comme lesbienne ou homosexuelle. Bien souvent, c'est la rencontre avec une personne désignée comme telle qui permet de se définir. Il en découle, la plupart du temps, une réévaluation de soi en tant que «femme». Pour beaucoup des interviewées, la nouvelle nomination de soi leur a permis «d'assumer» un genre autre que celui qui leur était assigné en tant que «femme». Autre exemple : si l'on prend l'espace public (la rue, les transports publics, etc.), on sait que ce dernier n'est pas neutre. Il est régi, d'une part, par une norme hétérosexuelle, mais aussi, par une norme androcentrée : codifié par les hommes, il contribue à modifier le comportement des femmes.

MB : Et tu appliques aussi cette critique de la neutralité au chercheur?

NC : Oui. Tout comme sur le terrain, je pense qu'on ne peut pas oblitérer la place que l'on y occupe selon le statut social de sexe et de genre (entre autres rapports sociaux). Par exemple, j'ai participé, il y a quelques mois à l'enquête sur la sexualité (INED, INSERM) et j'ai interrogé des femmes et des hommes. Il était évident que, selon que j'interrogeais des femmes ou des hommes, nos rapports n'étaient pas les mêmes, du fait que j'étais une femme sur ce terrain (sans que mes interlocuteurs sachent si j'étais lesbienne ou hétérosexuelle). Je crois qu'il est important d'avoir cela en tête pour analyser des données et pratiquer un terrain, c'est ce que l'on pourrait appeler brièvement les savoirs situés.

MB : Je rejoins ce que tu dis. En même temps, si sur le plan théorique cela peut paraître évident, cela reste difficile à analyser. Quelle(s) dimension(s) - de sexe, de classe, d'âge, etc. - jouent et comment se combinent-elles dans la trajectoire comme dans l'interaction ? Ce n'est pas si simple car on ne peut ni isoler ces facteurs ni les agréger d'où la critique de l'intersectionalité [L'intersectionalité renvoie à l'appréhension croisée des rapports de pouvoir]. En effet, à la suite des acquis du Black Feminism, la pertinence d'un sujet politique féminin qui renverrait à un «nous les femmes », fondé sur une expérience commune des rapports de domination et apte à légitimer et à faire advenir une mobilisation universelle a été posée. Ainsi, le Black Feminism vise d'une part cette tendance du féminisme à confondre la situation de certaines femmes avec la situation de toutes les femmes et d'autre part la condescendance de ces femmes dont la posture consiste à dire « je sais mieux que vous ce qui vous caractérise »; ce qui pose la question de qui est légitime pour parler au nom de qui. 
Chandra Mohanty reproche à la recherche féministe occidentale de créer l'image d'une femme $d u$ tiers-monde, victime sans capacité d'autonomisation, opprimée par la famille, la culture et la religion. Ces études «colonisent toute l'hétérogénéité matérielle et historique de la vie des femmes du tiers-monde » pour construire l'image unique d'une femme qui «mène une vie essentiellement limitée par son appartenance au genre féminin (lire : sexuellement inhibée) et au tiers-monde (lire : ignorante, pauvre, sans éducation, liée par la tradition, confinée dans ses foyers, tournée vers la famille, victimisée, etc.), par opposition à la femme occidentale libérée » $(1991,56)$.

Cependant, les rapports de pouvoir ne sont pas linéaires et cette conception d'une intersection du sexisme, du racisme et des rapports de classe peine à rendre compte des rapports sociaux dans leur dynamique et leur complexité. Paola Baccheta, par exemple lui préfère le terme de co-formation (2009). Par co-formation, elle entend « la dynamique par laquelle des pouvoirs (qui peuvent être visibles ou non, comme le genre, la sexualité, le racisme, les rapports sociaux de classe, la colonialité, etc.), effectuant des micro-circulations, se forment de manière indissociable, se transforment syncrétiquement et opèrent de concert pour produire tous les sujets, les conditions matérielles, les conduites et les objets existant » (Baccheta, 2009). Cette notion met en relief les inséparabilités de pouvoirs précédemment conceptualisés comme analytiquement distincts (le genre, la sexualité, le racisme, les classes sociales entre autres). Paola Baccheta applique cette notion à un groupe de lesbiens " of color », le Groupe $d u 6$ novembre, ce qui permet par exemple de penser le fait que des lesbiennes musulmanes portent le voile.

NC : Tu n'as pas répondu à la question!

MB : Oui... En fait, ces questions demeurent encore en suspens à la fois sur le plan de mes recherches empiriques à travers la question des géographies lesbiennes et sur le plan épistémologique. C'est d'ailleurs toute la question du séminaire La dimension sexuée du processus d'enquête : genre, sexualité et réflexivité que je co-anime avec Sébastien Roux à l'EHESS.

Pourquoi assiste-t-on aujourd'hui à un manque d'articulation dans les travaux sur l'homosexualité, tant en géographie qu'en sociologie, entre le genre et la sexualité et, audelà, des autres rapports de pouvoir : classe, race et âge?

NC : À mon sens, cela dénote d'une dépolitisation des sujets autour des sexualités. En fait, bien souvent la sexualité sert de support analytique d'expérience sans qu'elle soit pour autant contextualisée dans un rapport social plus large, tel que le genre, et bien pire la classe sociale, la «race», etc. Les travaux ayant articulé ces deux dernières dimensions, même dans la génération actuelle, émanent bien souvent d'auteurs critiques ou se réclamant d'une certaine posture féministe des théories de l'intersectionnalité.

MB : Je partage aussi ton analyse d'un point de vue politique et épistémologique. À cela s'ajoute une autre dimension plus pragmatique. Au plan théorique, certains auteurs ont affirmé qu'il y avait un effet cumulatif des positions hiérarchiques dans l'espace social (être une femme noire lesbienne issue d'origine populaire...), alors que d'autres ont mis en relief les interactions qu'il y avait entre ces dimensions produisant parfois des effets émancipateurs (c'est justement l'expérience du racisme qui a permis à certaines femmes de prendre conscience de celle du sexisme). Ce n'est donc pas une question simple que 1'on peut traiter par un simple cumul d'informations, en multipliant les cases lors d'une enquête par exemple. 
C'est pourquoi une approche biographique des trajectoires me semble plus intéressante pour aborder ces questions que la seule prise en compte des différents critères de l'âge, du sexe, de la classe, etc.

\section{Parcours académique et résistances autour d'un objet}

\section{Comment notre travail a-t-il été accueilli dans nos deux disciplines?} [question suggérée par les évaluateurs] Et quelles ont été vos stratégies de publication (revues spécifiques ou généralistes) et quels sont les problèmes que vous avez rencontrés en la matière?

MB : Pour ma part, en géographie, l'accueil s'est fait de façon très contrastée, avec des évolutions liées à l'avancement de ma thèse et à mon insertion professionnelle au sein de l'université. Je crois que certains avaient besoin de gages, montrant d'une part, que ce type d'études pouvait s'insérer dans la discipline en réponse à la fameuse question : «Est-ce que c'est de la géographie et pas de la sociologie ?» et, d'autre part, que ce type d'études n'était pas totalement fantaisiste mais s'appuyait sur des méthodes éprouvées d'enquête. Ce type de sujet implique d'apporter constamment des garanties de rigueur, pas seulement pour soi mais aussi pour ceux qui suivent. C'est important « d'asseoir le champ ».

Je dois aussi remarquer que si ce sujet a suscité des résistances, il a aussi bénéficié d'importants soutiens, parmi lesquels l'École Doctorale de Géographie [sous la forme d'une allocation de recherche] et de nombreux chercheurs, à commencer par mon directeur de thèse, Christian Grataloup, mais aussi de François Durand-Dastès, de France Guérin-Pace, de Catherine Rhein et de nombreux membres de l'UMR-Géographie-cités qui m'ont beaucoup soutenu tout au long de mes recherches.

NC : Pour ma part, en sociologie, mon travail a été bien accueilli, si j'en crois les offres de publication et de participation à des colloques ou à des séminaires, toutefois on ne pourra vraiment le vérifier qu'à l'aune de l'obtention d'un poste. En ce qui concerne la question des publications, je n'ai pas usé spécialement de stratégies. Mais il est vrai qu'au début de mes recherches jusqu'à la fin de la thèse, je n'ai pas proposé de publications d'articles à des revues dites de rang $\mathrm{A}$ ou plus généralistes. Inséré dans un milieu académique féministe, j'ai davantage publié dans des revues ou des collections spécialisées. Toutefois des contacts avec des chercheurs émanant de revues plus généralistes comme la revue interdisciplinaire Corps m'ont permis une plus large diffusion de certains de mes travaux. Mais en ce qui concerne mon parcours de doctorat, j'ai fait ma thèse sans aucun financement, ce qui est révélateur de la très faible prise en compte institutionnelle de l'objet d'étude qu'est le lesbianisme. Aujourd'hui, je crois que les choses sont en train d'évoluer, si l'on en croit les ouvertures de financement que ce sujet, entre autres dans le champ de la santé. Quand aux personnes qui m'ont soutenu, bien sûr il y a eu d'abord ma directrice de thèse : Marie-Elisabeth Handman (LAS/EHESS) car sans elle je n'aurais jamais pu m'inscrire en thèse, ainsi que Michel Bozon (INED), mais il y eu aussi et surtout de nombreuses chercheurs et théoriciennes issus des savoirs féministes. Je pense particulièrement à Michèle Ferrand, Nicole-Claude Mathieu, Hélène Rouch, Claire Michard, Brigitte Lhomond, Dominique Fougeyrollas, Laurence Tain, Florence Binard, etc.

MB : Pour ce qui est des publications, je n'ai pas à proprement parler mis en œuvre de stratégie. Il faut dire que j'ai peu publié et que beaucoup de publications sont le fruit de rencontres et de hasards. La stratégie éditoriale à laquelle je me suis associée a davantage été 
la création et le développement de la revue pluridisciplinaire Genre, sexualité \& société qui vise à légitimer ces objets dans le champ académique.

[question suggérée par les évaluateurs] Des séjours à l'étranger ont-ils joué un rôle dans vos parcours?

NC : Je ne peux pas encore témoigner sur de tels échanges car jusqu'à présent je n'ai pas fait ce que l'on pourrait appeler des séjours à l'étranger, je me suis surtout déplacée dans des colloques internationaux où j'ai pu par contre établir un réseau de connaissances et d'échanges sur les recherches produites dans divers pays. Ces déplacements m'ont permis de rencontrer des chercheurs (chercheuses la plupart du temps) travaillant sur le lesbianisme, ce qui est par contre très intéressant sur le plan du déploiement des connaissances sur le sujet.

MB : Même situation de mon côté. Je devais partir aux États-Unis travailler de façon comparative, mais cela ne s'est pas fait. Après la thèse, les colloques internationaux notamment le meeting annuel de l'Association of American Geographers ou celui de la Royal Geographical Society ont été des lieux de socialisation importants à la fois en terme de confrontation scientifique, de découverte de l'actualité de ces questions et de constitution de collaborations internationales. Néanmoins, je ne regrette pas que ces échanges aient eu lieu plus tardivement dans mon parcours car le décalage entre la France et la Grande-Bretagne est tel que j'aurai eu des difficultés à mener des recherches autonomes, j'aurais certainement reproduit ce que faisait les géographes britanniques sans tenir compte des apports d'autres disciplines comme la sociologie, l'histoire ou l'anthropologie.

Le travail de dé/catégorisation produit par les travaux sur l'homosexualité a-t-il selon vous produit des modifications dans le champ disciplinaire des études sur la sexualité ?

NC : Oui, dans le prolongement de la sociologie interactionniste (notamment celle de la déviance) qui a contribué à la déconstruction des catégories du sens commun, les travaux portant sur l'homosexualité ont permis un renouvellement des objets, conduisant à ne plus considérer comme un acquis des données comme le sexe, le genre, la « race », etc. En outre, il me semble qu'au-delà même des disciplines internes au champ de la recherche, la question de la norme hétérosexuelle est aujourd'hui devenue recevable. Le fait même que l'on entende parler d'hétérosexualité dans l'univers médiatique peut être un indicateur de changement. Toutefois, on pourra percevoir une évolution sensible quand des postes seront fléchés en études de genre par exemple, incluant les feminist studies, ou les queer studies comme aux États-Unis. Car, si en France, on se dirige vers un système libéral en ce qui concerne le fonctionnement des universités, il faudrait au moins en avoir quelques avantages, autrement dit que ce soit la demande qui fasse plier l'offre et non le contraire.

MB : Je ne partage pas tout à fait ton optimiste et je crois qu'il faut y regarder de plus près. En particulier, qui sont les chercheurs pour qui l'hétérosexualité est une norme et une construction socio-historique ? Je n'ai pas l'impression que les hétérosexuels soient très nombreux parmi eux. Ensuite, parmi ces chercheurs, combien réifient dans leurs travaux les catégories qu'ils mobilisent ou des oppositions binaires comme ville/campagne, ville centre/banlieue, eux/nous ? Ce que certains dénaturalisent d'un côté, ils l'essentialisent de l'autre. Donc effectivement, des avancées ont été faites, mais il me semble qu'il reste beaucoup à faire, notamment dans un dialogue et une écoute réciproque des chercheurs, toutes disciplines et sexualités confondues, pour faire front commun sur ces questions. 
[question suggérée par les évaluateurs] Quelle est votre expérience de l'enseignement ? Vis-àvis des étudiant-e-s et des collègues avez-vous éprouvé de la difficulté à les convaincre de la pertinence de cours dédiés à la sexualité dans les deux disciplines?

NC : Pour ma part, il m'est difficile de répondre à la question, n'ayant pas pour l'instant de poste, je ne suis pas véritablement insérée dans un programme d'enseignement. Par contre, en dehors des charges de cours, où $\mathrm{j}$ 'enseigne de la sociologie généraliste : méthodologie qualitative, ou initiation à la recherche, etc., j'ai également dispensé des cours sur des formations fléchées en études genre en tant qu'ATER à 1'Université Paris 7 Denis-Diderot, et dans un master genre à l'Université de sciences politiques de Reims, qui aujourd'hui n'existe plus. Il était évident que dans ces cours l'articulation genre et sexualité ne posaient pas de problème, elle était même réclamée par les étudiant-e-s et les collègues.

MB : Tu oublies le cours de master sur la sexualité à l'IDUP à l'automne dernier avec cet étudiant qui manifestait ostensiblement son désintéressement et qui a fini par dire qu'il désapprouvait ce type d'explicitation des rapports de pouvoir articulant genre et sexualité que cela devait «rester de l'ordre de l'intime», "ne pas se dire » et qu' « il ne voulait pas savoir ». Tu as réussi à lui prouver que cela dépasser très largement le cadre des pratiques sexuelles. Cette anecdote est assez révélatrice des résistances que l'on rencontre. Elles sont d'autant plus difficiles à désamorcer que d'une part elles ne s'expriment pas toujours et que d'autre part le volume global consacré à ce type d'enseignement est très insuffisant pour mettre en place un discours critique. Parmi les rapports de domination, les rapports sociaux de sexe sont parmi les plus difficiles à déconstruire tant ils sont intériorisés et naturalisés. Je suis toujours surprise d'être interpellée par des étudiantes qui prennent la défense de leurs camarades, se sentent « agressées » par un discours qu'elles refusent au motif qu'elles ne sont pas « victimes ».

NC : Oui, tu as raison.

Quelles sont les principales difficultés liées au choix d'objets tels que l'homosexualité ou le lesbianisme?

NC : La construction de la carrière professionnelle titularisable.

MB : J'aurais dû mal à faire la même réponse, ayant eu la chance de trouver un poste à l'issue de ma thèse. Je dirais «la gestion du soupçon », qui fait que n'étant pas insider [en tant que femme hétérosexuelle], il demeure un soupçon de la part de certains membres du groupe dans le champ académique [cette situation ne s'est jamais produite sur le terrain]. C'est assez instructif en matière de rapports sociaux de sexe, d'être systématiquement renvoyée par quelques chercheurs gays à ma condition de dominante, parce qu'hétérosexuelle, selon un registre et des modalités de disqualification qui relève d'un rapport de pouvoir qu'ils n'emploieraient jamais avec un chercheur homme. Cela crée une situation inconfortable dans laquelle, à la fois, vous endossez une partie du stigmate en portant l'objet dans le champ académique, néanmoins vous ne pouvez pas revendiquer légitimement la reconnaissance et les formes de solidarité que cela implique parmi les membres du groupe stigmatisé. Ajoutez à cela le fait que de nombreux chercheurs travaillant sur ce sujet depuis de nombreuses années n'ont pas eu de postes, cela crée un fort ressentiment. Être l'outsider qui engrange les bénéfices d'un travail sur le groupe, c'est la position idéale pour cristalliser les frustrations. C'est d'autant plus dommageable que c'est une façon de se tromper d'ennemi ; c'est jouer le jeu de la division et ne pas s'en prendre aux sources du problème à savoir la reconnaissance 
des études sur le genre et la sexualité en France et la politique libérale de la recherche menée par le gouvernement.

\section{Références bibliographiques}

Adler Sy, Brenner Johanna, 1992, «Gender and Space: Lesbians and Gay Men in the City », International Journal of Urban and Regional Research, vol.16, ${ }^{\circ} 1$, p. 24-34.

Bacchetta Paola, 2009, «Co-Formations : des spatialités de résistance décoloniales chez les lesbiennes "of color" en France ", Genre, sexualité \& société, $\mathrm{n}^{\circ} 1$ (http://gss.revues.org/index810.html).

Barthe Francine, Hancock Claire (dir.), 2005, «Le genre. Constructions spatiales et culturelles », Géographie et cultures, n54, L’Harmattan, 2005.

Becker Howard S., 1985, Outsiders. Études de sociologie de la déviance, Paris, Métailié.

Binard Florence, 2003, Les discours entourant l'homosexualité féminine dans l'entre-deux guerres en Grande-Bretagne, thèse de doctorat sous la direction de Françoise Barret-Ducrocq, Paris, Université Paris 7.

Blidon Marianne, 2007, Distance et rencontre. Éléments pour une géographie des homosexualités, thèse de doctorat sous la direction de Christian Grataloup, Paris, Université Paris 7-Denis Diderot.

Blidon Marianne, 2008a, «Jalons pour une géographie des homosexualités », L'Espace géographique, $\mathrm{n}^{\circ} 2$, p. 175-189.

Blidon Marianne, 2008b, «La casuistique du baiser. L'espace public, un espace hétéronormatif », Echogéo, n 5 (http://echogeo.revues.org/index5383.html).

Blidon Marianne, 2009, «La Gay Pride entre subversion et banalisation », Espace, Populations, Sociétés, n², p. 305-318.

Boehringer Sandra, 2007, L'Homosexualité féminine dans l'Antiquité grecque et romaine, Paris, Belles Lettres, Collection d'études anciennes, 2007.

Boivin Nicolas (dir.), 2008, «Sexe de l'espace, sexe dans l'espace », Les cahiers de l'ADES, $\mathrm{n}^{\circ} 2$ (http://www.ades.cnrs.fr/spip.php?article492).

Bondi Liz, 1993, "Gender and geography, crossing boundaries », Progress in Human Geography, vol. 17, n 2, p. 241-246.

Bonnet Marie-Jo, 1981, Un choix sans équivoque. Recherches historiques sur les relations amoureuses entre les femmes, $X V I^{e}-X X^{e}$ siècle, Paris, Denoël.

Bonta Mark, Protevi John, 2004, Deleuze and Geophilosophy: A Guide and Glossary, Edinburgh, Edinburgh University Press.

Butler Judith, 2005, Trouble dans le genre, Paris, La découverte.

Chetcuti Natacha, Michard Claire (dir.), 2003, Lesbianisme et féminisme : histoires politiques, Paris, L'harmattan.

Chetcuti Natacha, Jaspard Maryse (dir.), 2007, Violences envers les femmes. Trois pas en avant deux pas en arrière, Paris, L'Harmattan, 2007. 
Chetcuti Natacha, 2008, Normes socio-sexuelles et lesbianisme : Définition de soi, catégories de sexe/genre et script sexuel, thèse de doctorat sous la direction de Marie-Elisabeth Handman, Paris, EHESS.

Chetcuti Natacha, 2009, «De "On ne naît pas femme" à "On n'est pas femme". De Simone de Beauvoir à Monique Wittig», Genre, sexualité \& société, $\mathrm{n}^{\circ} 1$ (http://gss.revues.org/index477.html).

Chetcuti Natacha, 2010, Vies lesbiennes : Représentations de soi, couples et sexualité, Paris, Payot (à paraître en octobre 2010).

Chivallon Christine, 2001, «Les géographies féministes. Un plaidoyer convaincant pour la constitution de connaissances "situées" ", in Géographies anglo-saxonnes, Staszak JeanFrançois et ali, Paris, Belin, p. 57-63.

Collignon Béatrice, Staszak Jean-François, 2004, «Que faire de la géographie postmoderniste ? », L'Espace géographique, vol. 33, n 1 , p. 38-42.

Coulmont Baptiste, 2003, «Géographie de l'Union civile au Vermont». Mappemonde, $\mathrm{n}^{\circ} 71$, p. 13-18.

Creton Dominique (dir.), 2002, « Questions de genre », Espace, populations, sociétés, Lille.

Creton Dominique (dir.), 2004, «Espace, genre et sociétés », Espace, populations, sociétés, Lille.

Decroly Jean-Michel et ali, 2006, «Les territoires de l'homosexualité à Bruxelles : visibles et invisibles». Cahiers de géographie du Québec, n¹40, p. 135-150.

Descoutures Virginie, 2008, Les mères lesbiennes : contribution à la sociologie de la parenté, thèse de doctorat sous la direction de François de Singly, Paris, Université Paris Descartes.

Fall Juliet, 2005, «Michel Foucault and Francophone geography», EspacesTemps.net (http://espacestemps.net/document1540.html).

Fernández Víctor Salinas, 2007, «Comunidad Gay y Espacio en España », Boletín de la Asociación de Geógrafos Españoles , nº 43, p. 241-260.

Gagnon John H., Simon William, 1973, Sexual Conduct. The Social Sources of Human Sexuality, Chicago, Aldine.

Giraud Colin, 2009, «Les commerces gays et le processus de gentrification », Métropoles, 5 (http://metropoles.revues.org/3858).

Goonewardena Kanishka, Kipfer Stefan, Milgrom Richard, Schmid Christian (eds), 2008, Space, Difference, Everyday Life: Reading Henri Lefebvre, London, Routledge.

Grésillon Boris, 2000, " "Faces cachées de l'urbain" ou éléments d'une nouvelle centralité ? Les lieux de la culture homosexuelle à Berlin », L'espace géographique, n²9, p. 301-313.

Guillaumin Colette, 1992, Sexe, race et pratique du pouvoir, Paris, Côté-femmes.

Guillaumin Colette, 2002, L'idéologie raciste, genèse et langage actuel, Paris, Gallimard.

Jaurand Emmanuel, 2005, «Territoires de mauvais genre ? Les plages gays », Géographie et cultures, $\mathrm{n}^{\circ} 54$, p. 71-84.

Lesselier Claudie, 1987, Aspects de l'expérience lesbienne en France, 1930-1968, DEA de sociologie sous la direction de Robert Castel, Université Paris 7, novembre 1987 (non publié). 
Leroy Stéphane, 2005, «Le Paris gay. Eléments pour une géographie de l'homosexualité », Annales de géographie, $\mathrm{n}^{\circ} 646$, p. 579-601.

Lhomond Brigitte, 1987, «Entre l'homme et la femme, le personnage de la lesbienne », communication au colloque international Homosexuality, which Homosexuality ?, Amsterdam, 15-18 décembre 1987 [publiée dans Les Lesbianaires, n 20-21, février 1988, Centre de documentation et de recherches sur le lesbianisme radical, p. 53-59, publiée en anglais, "Between Man and Woman: the Character of the Lesbian », Journal of Homosexuality, vol. 25, ${ }^{\circ}$ 1/2, Haworth Press, 1993, p. 63-73].

Lhomond Brigitte, 1997, «Le sens de la mesure. Le nombre d'homosexuel/les dans les enquêtes sur les comportements sexuels et le statut de groupe minoritaire », Sociologie et sociétés, vol. 29, $\mathrm{n}^{\circ} 1$, p. 61-69.

Lieber Marylène, 2008, Genre, violences et espaces publics. La vulnérabilité des femmes en question, Paris, Les presses de SciencesPo.

Massey Doreen, 1994, «A global sense of place; "A placed called home?” », in Space, place and gender, Oxford, Blackwell, p. 146-173.

Massey Doreen, 1995, «The conceptualization of place », in Massey D., Jess P. (eds), A place in the world? Place, Culture and Globalization, Oxford, Oxford University Press, p. 4585.

McDowell Linda, 1993, «Space, place and gender relations », Progress in Human Geography, vol. 17, $\mathrm{n}^{\circ} 2$.

McDowell Linda, Sharp Joanne (eds), 1999, A feminist glossary of human geography, London, Arnold.

Mathieu Nicole-Claude, 1991, L'anatomie politique. Catégorisationset idéologies du sexe, Paris, Côté-femmes, p. 227-266.

Mendes-Leite Rommel, Busscher Pierre-Olivier (de), 1997, Back-rooms. Microgéographie sexographique de deux back-rooms parisiennes: appropriation de l'espace corporel et gestion de la sexualité face au VIH, GKC, Lille.

Mendes-Leite Rommel, Proth Bruno, 1998, «L'itinéraire des désirs. Déambulations masculines sur les lieux de drague à Paris », French Cultural Studies, n ${ }^{\circ}$ X, p. 367-383.

Mohanty Chandra Talpade, 1991, «Under Western Eyes: Feminist Scholarship and Colonial Discourse », in C. Mohanty, A. Russo, L. Torres (dir.) Third World Women and the Politics of Feminism, Bloomington, Indiana University Press.

Podmore Julie, 2006, "Gone "underground"? Lesbian visibility and the consolidation of queer space in Montréal », Social and Cultural Geography, vol.7, n, p. 595-625.

Pollak Michael, 1981, «Les vertus de la banalité », Le débat, ${ }^{\circ}{ }^{10}$, p. 132-143.

Proth Bruno, 2002, Lieux de drague, scènes et coulisses d'une sexualité masculine, éditions Octares, Toulouse.

Raffestin Claude, 1997, « Foucault aurait-il pu révolutionner la géographie? », in Au risque de Foucault, Paris, Centre Georges Pompidou, p. 141-149.

Redoutey Emmanuel, 2002, «Géographie de l'homosexualité à Paris, 1984-2000 », Urbanisme, ${ }^{\circ} 325$, p. 59-63.

Séchet Raymonde, Veschambre Vincent (dir.), 2006, Penser et faire la géographie sociale. Contributions à une épistémologie de la géographie sociale, Rennes, PUR, 2006. 
Sibalis Michael, 1995, «Paris », Queer Sites. Gay urban histories since 1600, Routledge, New York, p. 10-37.

Sibalis Michael, 2001, «Les espaces des homosexuels dans le Paris d'avant Haussmann », La modernité avant Haussmann. Formes de l'espace urbain à Paris 1801-1853, Editions Recherches, Paris, p. 231-241.

Tabet Paola, 2004, La grande arnaque. Sexualité des femmes et échange économico-sexuel, Paris, L'Harmattan.

Tin Louis-Georges, 2003, «Hétérosexisme », in Dictionnaire de l'homophobie, Paris, PUR, p. 207-211.

Wittig Monique, 1980a, « On ne naît pas femme », Questions féministes, n ${ }^{\circ}$, p. 75-84.

Wittig Monique, 1980b, «La pensée straight », Questions féministes, nº 7, p. 45-53. 